\title{
A Metaheuristic Approach for Parameter Fitting in Digital Spiking Silicon Neuron Model
}

\author{
Takuya Nanami \\ The University of Tokyo, Institute of industrial Science \\ Tokyo, Japan \\ Filippo Grassia \\ LTI Lab., University of Picardie Jules Verne \\ Saint-Quentin, France \\ Takashi Kohno \\ The University of Tokyo, Institute of industrial Science \\ Tokyo, Japan \\ nanami@sat.t.u-tokyo.ac.jp,filippo.grassia@u-picardie.fr,kohno@sat.t.u-tokyo.ac.jp
}

\begin{abstract}
DSSN model is a qualitative neuronal model designed for efficient implementation in digital arithmetic circuit. In our previous studies, we developed automatic parameter fitting method using the differential evolution algorithm for regular and fast spiking neuron classes. In this work, we extended the method to cover low-threshold spiking and intrinsically bursting. We optimized parameters of the DSSN model in order to reproduce the reference ionicconductance model.
\end{abstract}

Keywords: Spiking neuron model, Low-threshold spiking, Intrinsically bursting, Differential evolution, FPGA

\section{Introduction}

The silicon neuronal network is composed of analog and (or) digital circuits that solve the differential equations of neuronal and synaptic models. Different kinds of neuronal models have been used in silicon neuronal networks because of the trade-off between reproducibility of neuronal activities and computational efficiency. For example, Merolla et al. ${ }^{1}$ constructed a digital silicon neuronal network comprising 1 million spiking neurons and 256 million synapses. They adopted Leaky integrate-and-fire (LIF) model that is one of the most commonly used and simple neuron models. Thomas et al. ${ }^{2}$ implemented 1024-spiking neurons of Izhikevich (IZH) model $^{3}$ on a fieldprogrammable gate array (FPGA). Those LIF, expanded LIF, and IZH models are categorized as integrate-and- fire (I\&F) based model which approximates a spiking process by the resetting of the state variables. The I\&Fbased models can be implemented resource-efficiently, but are capable of reduced reproducibility of complex neuronal activities. For example, these models assume that the amplitude of spikes is fixed, whereas the spike intensity is nonuniform in the nervous system. ${ }^{4}$

We have been studying qualitative neuronal models for digital as well as analog circuit implementation that satisfy both the reproducibility of neuronal activities and low computational cost. 5, 6, 7,8 The core idea of our qualitative-modeling-based approach is to reproduce the core mathematical structures that a wide variety of neuronal activities. In our previous studies, 9,10 we extended the DSSN models ${ }^{7}$ to support various neuronal classes; regular spiking (RS), fast spiking (FS), intrinsically bursting (IB), low-threshold spiking (LTS), 
elliptic bursting (EB), and parabolic bursting (PB). We also studied an automatic parameter fitting method using the differential evolution (DE) algorithm ${ }^{11}$ for the RS and FS classes. ${ }^{12}$

In this work, we developed the method for the lowthreshold spiking and intrinsically bursting classes. Parameters were optimized to reproduce waveforms of the ionic-conductance models in Ref. 13 and reduce the circuit resource requirements for implementation.

The remainder of this paper is organized as follows. Section 2 introduces our neuron model, the DE algorithm, and details of our parameter optimization procedure. The result is shown in Section 3. Section 4 summarizes this work and suggests ideas for the future.

\section{Method}

\subsection{Digital Spiking Silicon Neuron model}

The 4-variable DSSN model is a qualitative neuron model that can simulate various neuronal classes (RS, FS, IB, LTS, EB, and PB) by Euler's method with fixed point operation. Its equations are given by

$$
\begin{gathered}
\frac{d v}{d t}=\frac{\emptyset(u)}{\tau}\left(f(v)-n-q+I_{0}+I_{\text {stim }}\right), \\
\frac{d n}{d t}=\frac{1}{\tau}(g(v)-n), \\
\frac{d q}{d t}=\frac{\varepsilon}{\tau}(h(v)-q), \\
\frac{d u}{d t}=\frac{\varepsilon_{u}}{\tau}\left(v-v_{u}-\alpha_{u} u\right), \\
\varnothing(\mathrm{u})= \begin{cases}\emptyset_{0} & \left(u<r_{u 0}\right) \\
\emptyset_{1} & \left(r_{u 0}<u<r_{u 1}\right) \\
\emptyset_{2} & \left(r_{u 1}<u\right),\end{cases} \\
f(v) \equiv \begin{cases}a_{f n}\left(v-b_{f n}\right)^{2}+c_{f n} & (v<0) \\
a_{f p}\left(v-b_{f p}\right)^{2}+c_{f p} & (v \geq 0),\end{cases} \\
g(v) \equiv \begin{cases}a_{g n}\left(v-b_{g n}\right)^{2}+c_{g n} & \left(v<r_{g}\right) \\
a_{g p}\left(v-b_{g p}\right)^{2}+c_{g p} & \left(v \geq r_{g}\right),\end{cases} \\
h(v) \equiv \begin{cases}a_{h n}\left(v-b_{h n}\right)^{2}+c_{h n} & \left(v<r_{h}\right) \\
a_{h p}\left(v-b_{h p}\right)^{2}+c_{h p} & \left(v \geq r_{h}\right),\end{cases}
\end{gathered}
$$

where $v$ corresponds to the membrane potential. Variables $n$ and $q$ are the fast and slow variables which abstractly describe the activity of the fast and slow ion channels, respectively. Variable $u$ is the slowest variable that modifies the structure of the fast subsystem comprising $v$ and $n$, and used in only the IB mode. In the RS, FS, and LTS modes, $u$ is fixed. Parameter $I_{0}$ is a constant and $I_{\text {stim }}$ represents the input stimulus. Parameters $\emptyset_{0}, \emptyset_{1}, \emptyset_{2}, \varepsilon, \varepsilon_{u}$, and $\tau$ control the time constants of the variables. Parameters, $r_{g}, r_{h}, v_{u}, \alpha_{u} a_{x}$, $b_{x}$, and $c_{x}$, where $x$ is $f n, f p, g n, g p, h n$, or $h p$, are constants that adjust the shape of the $v-, n-, q$ - and $u$ nullclines. Parameters $r_{u 0}$ and $r_{u 1}$ are used to select $\emptyset$ depending on $u$. All of the variables and constants in this qualitative model are purely with no physical unit. Cubic shaped $v$-nullcline is a key to replicate the spiking dynamics. Because multipliers are resourceconsuming in a digital arithmetic circuit, the DSSN model adopts a piecewise quadratic function for the $v$ nullcline so that its numerical integration step requires only one multiplication between variables.

\subsection{Differential evolution algorithm}

The DE algorithm ${ }^{11}$ is a popular heuristic method to solve optimization problems using a real number function. It is one of the population-based optimization algorithms in which a number of parameter sets are converged to quasi-optimal solutions with which the DSSN model reproduces the target waveform. The initial random parameter sets have to be selected carefully so that the behavior of the DSSN model does not diverge. Detailed explanation is written in the previous study. ${ }^{12}$

\subsection{Parameter optimization procedure}

The LTS class neurons are found in the thalamus. It has the spike-frequency adaptation as with the RS class, and has the rebound bursting; neurons in the LTS class generate a burst firing just after the termination of a sufficiently strong and long hyperpolarizing stimulus. The IB class is a well-known bursting neuron class in the cortex. Neurons in the IB class generate a burst firing immediately at the onset of a sufficiently strong stimulus, then continue spiking until its termination.

The DSSN model has 28 parameters, and exploring their spanning space is not realistic for the computers with average power. To avoid this problem, we divided the state variables of the DSSN model into two groups based on their time scale, and tuned each group in sequence. In our previous study, ${ }^{12}$ the RS and FS classes were characterized as the degree of spikefrequency adaptation, and we just optimized parameters of fast state variables in order to reproduce the saddle- 
(a)

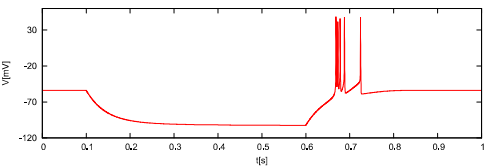

(b)

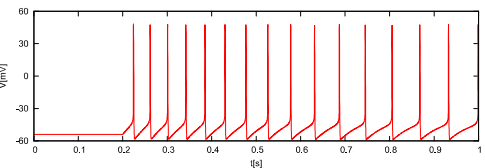

(c)

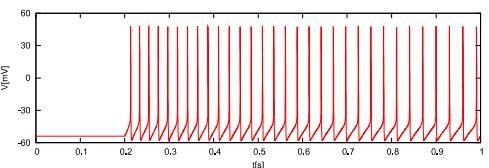

(d)

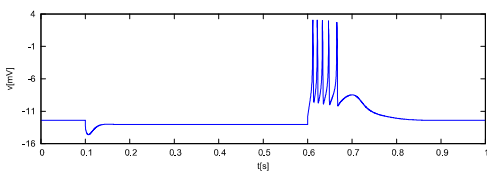

(e)

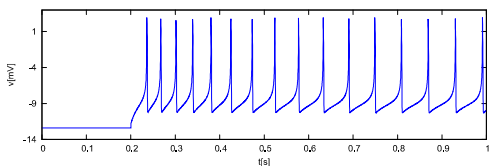

(f)

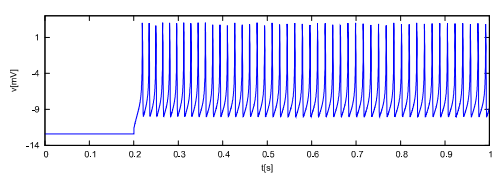

Fig. 1. Waveforms of the ionic-conductance model (red) and the DSSN model (blue) of the LTS class in response to an inhibitory (top) and weak excitatory (middle) and strong excitatory (bottom) step stimulus input.

node on invariant circle bifurcation. However, LTS and IB classes have more complex structure in their fasts subsystem. For the LTS class, we firstly explored the parameter group responsible for the dynamics of the fast state variables $v$ and $n$ so that the fast subsystem of our model is consistent with that of the ionic conductance model of the LTS class. Then, the parameters related to the slow state variable $q$ were determined so that the dynamical behavior of the whole ionic conductance model of the LTS class is reproduced. For the IB class, we firstly fitted the parameters responsible for the dynamics of the state variables $v, n$, and $q$ so that the dynamical behavior of the corresponding subsystem in the ionic conductance model of the IB class is reproduced. Then, the parameters related to the remaining variable $u$ were determined so that the dynamical behavior of the whole ionic conductance model of the IB class is reproduced. (a)

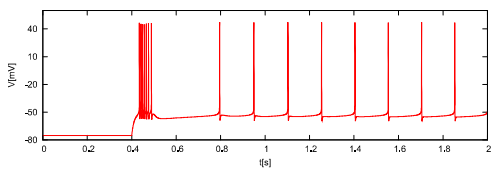

(b)

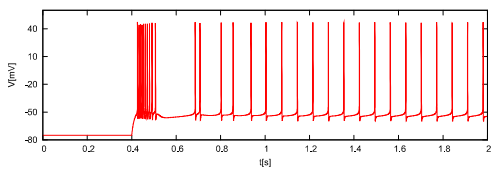

(c)

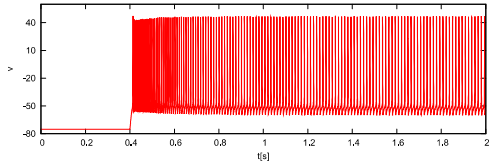

(d)

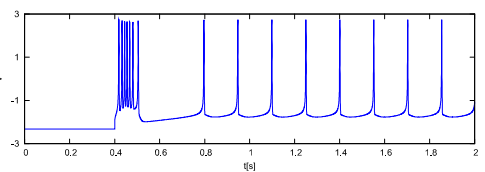

(e)

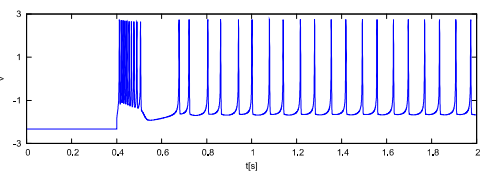

(f)

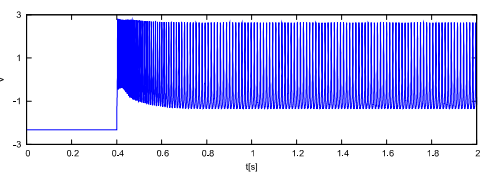

Fig. 2. Waveforms of the ionic-conductance model (red) and the DSSN model (blue) of the IB class in response to a weak (top) and middle (middle) and strong (bottom) excitatory step stimulus input.

In the DSSN model's circuit implementation, multiplication between a coefficient and a state variable was realized by shifters and adders, and we needed a larger number of adders and shifters as the number of digits with value 1 in the fixed point representation of the coefficient increases. Parameters were optimized for not only reproducing waveforms of the ionicconductance model but also reducing the circuit size. We defined the cost functions $f_{1}$ and $f_{2}$ as follows,

$$
\begin{aligned}
& f_{1}(x)=f_{w 1}(x)+k_{b 1} f_{b 1}(x), \\
& f_{2}(x)=f_{w 2}(x)+k_{b 2} f_{b 2}(x),
\end{aligned}
$$

where $f_{\mathrm{w}}(x)$ is the function that calculates cost from the target waveforms of the ionic conductance model and the DSSN model with parameter vector $x$, based on the difference of spike timing as well as the mean square error of the state variable corresponding the membrane potential. In this function, minimum and maximum values of waveforms are normalized to 0 and 1 for 
comparison. Function $f_{b}$ is a number of digits with value 1 in the binary fixed-point expression of the coefficients in $x$. Constant $k_{b}$ is to balance these two cost functions. Parameters responsible for the dynamics of the fast variables were determined to minimize the cost function $f_{1,2}$ using the $\mathrm{DE}$ algorithm.

In the LTS class, parameters related to state variables $v$ and $n$ were firstly determined to minimize the cost function $f_{1}$. Then the slow state variables are fixed in the ionic conductance model, which is used as the target waveforms in the $f_{w 1}$. Secondly, the remaining parameters related to the slow variable $q$ were determined to minimize the cost function $f_{2}$. The original ionic conductance model is used in the $f_{w 2}$. In the IB class, parameters related to state variables $v, n$, and $q$ were firstly determined to minimize the cost function $f_{1}$. Secondly, the remaining parameters related to the slowest variable $u$ were determined to minimize the cost function $f_{2}$.

\section{Result}

We found two parameter sets with which the DSSN model reproduces the activities of the ionic-conductance models for LTS and IB classes (Fig. 1). We evaluated the similarity of the spiking patterns between the ionicconductance model and the DSSN model by calculating some statistics while changing the stimulus intensity (Fig. 3). For the LTS class, we used $C_{V}$ and $L_{V}{ }^{14}$ and these are explained in our previous study. ${ }^{12}$ Our fitted model's $C_{V}-L_{V}$ characteristics are not completely the same as that of the reference model, but share the trend that $C_{V}$ and $L_{V}$ decreases as the stimulus intensity increases. For the IB class, we measured the number of spikes in the bursting duration and average firing rate after the bursting. With both models, the number of spikes in the bursting duration increases linearly as the average firing rate increases, although the exact value does not coincide completely. The similarity of these characteristics will be further estimated based on the requirements specified by the circuits' applications.

The results in Figs. 1, 2, and 3 were obtained by numerical integration with fixed-point operation and the Euler's method $\left(d t=2^{-13} \mathrm{~s}\right)$ in $C$ language. The same results are expected to be observed in the following FPGA implementation, because this condition is the same as our VHDL codes. We compiled the DSSN models for Xilinx XC7VX690T using Xilinx Vivado
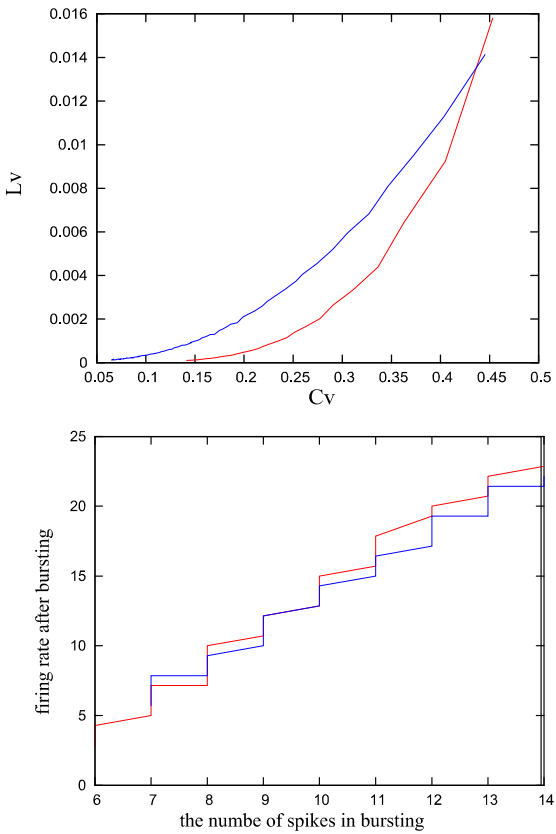

Fig. 3. Statistical properties of the ionic-conductance model (red) and the DSSN model (blue). In the LTS mode (left), the x-axis and y-axis are $C_{V}$ and $L_{V}$. In the IB mode (right), xaxis corresponds to the number of spikes in the bursting and $y$ axis corresponds firing rate after the bursting.

\begin{tabular}{|c|c|c|c|c|c|}
\hline \multicolumn{5}{|c|}{ Table. 1 : Device Utilization. } \\
\hline & LTS & IB & $\begin{array}{c}\text { LTS(hand- } \\
\text { fitted) }\end{array}$ & $\begin{array}{c}\text { IB(hand- } \\
\text { fitted) }\end{array}$ \\
\hline FF & 120 & 136 & 122 & 140 \\
\hline LUTs & 938 & 1265 & 1264 & 1639 \\
\hline DSPs & 1 & 1 & 1 & 1 \\
\hline
\end{tabular}

Design Suite. Device utilization is listed in Table 1. In the column labeled LTS (hand-fitted) and IB (handfitted), the resource usage for LTS and IB settings in Ref. 9 is shown. The requirement for LUTs was reduced without any loss of the reproducibility of the neuronal activities.

\section{Conclusion}

In this work, we extended the parameter optimization method for the DSSN model in the LTS and IB class modes. These classes have complex mathematical structures of the fast subsystem, thus we carefully designed our fitting procedure in order to reproduce important structures in these classes. We could find parameter sets with which the DSSN model reproduces the characteristics activities of LTS and IB classes using 
average-power PC ( a Xeon E5-2667 CPU with a NVIDIA P100 GPU ). Cost functions $f_{b x}$ were introduced to reduce the necessary circuit size. We confirmed that the DSSN model with determined parameter set can precisely reproduce the ionic conductance model in $\mathrm{C}$ simulation, and the necessary circuit size was simulated by Xilinx Vivado Design Suite. In our future work, we will apply our silicon neuronal networks to the spike pattern recognition. ${ }^{15}$

\section{Acknowledgement}

This work was partially supported by JSPS SAKURA Program and JST PRESTO and CREST

\section{References}

1. P. A. Merolla, J. V Arthur, R. Alvarez-Icaza, A. S. Cassidy, J. Sawada, F. Akopyan, B. L. Jackson, N. Imam, C. Guo, Y. Nakamura, B. Brezzo, I. Vo, S. K. Esser, R. Appuswamy, B. Taba, A. Amir, M. D. Flickner, W. P. Risk, R. Manohar, and D. S. Modha, "A million spikingneuron integrated circuit with a scalable communication network and interface", Science, Vol. 345, pp. 668-673, 2014

2. D. B. Thomas and W. Luk, "FPGA accelerated simulation of biologically plausible spiking neural networks," Proc. - IEEE Symp. F. Program. Cust. Comput. Mach. FCCM 2009, pp. 45-52, 2009.

3. E. M. Izhikevich, "Which model to use for cortical spiking neurons ?", IEEE Trans. Neural Netw. vol. 15, pp. 1063-1070, 2004

4. H. Alle and J. R. P. Geiger, "Combined analog and action potential coding in hippocampal mossy fibers", Science, vol. 311, pp. 1290-1293, 2006

5. T. Kohno and K. Aihara "A three-variable ultralowpower analog silicon neuron circuit" 2016 International Symposium on Nonlinear Theory and Its Applications, Japan, November 27th-30th, pp. 190-193, 2016

6. T. Kohno, M. Sekikawa, J. Li, T. Nanami, and K. Aihara, "Qualitative-Modeling-Based Silicon Neurons and Their Networks," Frontiers in Neuroscience, Vol. 10, No.273, pp. 1-16, Jun., 2016.

7. T. Kohno and K. Aihara. Digital spiking silicon neuron: Concept and behaviors in gj-coupled network. Proceedings of International Symposium on Artificial Life and Robotics 2007, 2007.

8. J. Li, Y. Katori, and T. Kohno, "An FPGA-based silicon neuronal network with selectable excitability silicon neurons," Frontiers in Neuroscience, Vol. 6, pp. 1-13, Dec., 2012.

9. T. Nanami and T. Kohno. "Simple cortical and thalamic neuron models for digital arithmetic circuit implementation", Frontiers in Neuroscience, Vol. 10, No.181, pp. 1-12, 2016

10. T. Nanami, K. Aihara, and T. Kohno, "Elliptic and parabolic bursting in a digital silicon neuron model", 2016 International Symposium on Nonlinear Theory and Its Applications, Japan, November 27th-30th, 2016

11. R. Storn, K. Price, "A Simple and Efficient Heuristic for global Optimization over Continuous Spaces", Journal of Global Optimization, Vol. 11, pp. 341-359, 1997.

12. T. Nanami, F. Grassia, and T. Kohno, "A parameter optimization method for Digital Spiking Silicon Neuron model", Journal of Robotics, Networking and Artificial Life, Vol. 4, pp. 97-101, 2017.

13. M. Pospischil, M. Toledo-Rodriguez, C. Monier, Z. Piwkowska, T. Bal, Y. Fregnac, H. Markram, and A. Destexhe. "Minimal Hodgkin-Huxley type models for different classes of cortical and thalamic neurons". Biological Cybernetics, Vol. 99, pp. 427-441, 2008.

14. S. Shinomoto, K. Shima, and J. Tanji, "Differences in spiking patterns among cortical neurons.," Neural Comput., vol. 15, no. 12, pp. 2823-2842, 2003.

15. T. Masquelier, R. Guyonneau, and S. J. Thorpe, "Competitive STDP-based spike pattern learning.," Neural Comput., vol. 21, pp. 1259-1276, 2009. 